\title{
Estimating the potential predictability of Western Australian surface temperature using monthly data
}
S. Grainger ${ }^{1}$
C. S. Frederiksen ${ }^{2}$
X. Zheng ${ }^{3}$

(Received 11 August 2008; revised 16 December 2008)

\begin{abstract}
The seasonal mean of a climate variable is considered to be a statistical random variable with two components: a slow component related to slowly varying (time scales of a season or more) forcings from external and internal atmospheric sources, and an intraseasonal component related to forcings from weather variability with time scales less than a season. Here, an extension of a previous Analysis of Variance method is proposed which deals with climate data in all seasons when estimating the intraseasonal variability. By removing this from the total variability, an estimate for the slow component, and hence the long range predictability, of the seasonal mean is made. The method is applied to monthly surface temperature data for Western Australia from 1951-2000.
\end{abstract}

http://anziamj . austms.org.au/ojs/index.php/ANZIAMJ/article/view/1414 gives this article, (c) Austral. Mathematical Soc. 2008. Published December 18, 2008. ISSN 1446-8735. (Print two pages per sheet of paper.) 


\section{Contents}

1 Introduction

C599

2 Methodology

C600

2.1 Statistical model . . . . . . . . . . . . . . C600

2.2 Solution for Australian surface temperature . . . . . . . C602

2.3 Potential predictability . . . . . . . . . . . . . C603

3 Potential predictability of Western Australian surface temperature

C604

4 Conclusions

C607

References

C608

\section{Introduction}

Climate variability has a major impact on the Australian economy, primarily through its direct impact on agriculture. Climate variability is managed through the provision of seasonal forecasts, the skill (or accuracy) of which is able to be assessed (for example, Fawcett et al. [1]). This raises the issue of where efforts and funds should be directed for better understanding the large scale processes which the provision of skilful forecasts depend upon. In particular, what are the regions and seasons where surface climate is potentially predictable?

Central to this question, and to seasonal forecasting, is the concept that the seasonal mean of a climate variable is considered to be a statistical random variable consisting of signal and noise components [2]. The signal is related to slowly varying (a season or more) boundary, or external, forcings or internal atmospheric variability and is considered as the 'slow' component 
of interannual variability of the seasonal mean [7]. The noise is related to internal atmospheric variability with time scales of about two weeks to a season and has been termed the 'intraseasonal' component of variability [7].

Frederiksen and Zheng [2] reviewed methods to estimate the intraseasonal variance. Recently, a method has been formulated to estimate the intraseasonal variance from monthly mean observations [9]. However, while the assumptions used are probably valid for summer and winter, this is not necessarily the case in all seasons. Here, we apply less restrictive assumptions about the monthly statistics of climate anomalies, in order to extend the analysis to include all seasons.

\section{Methodology}

\section{$2.1 \quad$ Statistical model}

Given the statistical model described above, the monthly mean anomaly of a climate variable $x$ is $[2,7,9]$

$$
x_{y m}=\mu_{y}+\epsilon_{y m}
$$

where $m(m=1,2,3)$ is the month index in the season, $y(y=1, \ldots, Y)$ is the year index and $Y$ is the total number of years, $\mu_{y}$ is the seasonal population mean, and $\epsilon_{y m}$ is the monthly departure of $x_{y m}$ from $\mu_{y}$. It is assumed that the vector $\left(\epsilon_{y 1}, \epsilon_{y 2}, \epsilon_{y 3}\right)$ is a stationary and independent random vector with respect to year $y$ [5]. Although $\mu_{y}$ and $\epsilon_{y m}$ are not able to be directly separated, it is possible to do so for their respective variances $\mathrm{V}$ [9]

$$
V\left(x_{y o}\right)=V\left(\epsilon_{y o}\right)+V\left(\mu_{y}\right)+2 V\left(\mu_{y}, \epsilon_{y o}\right)
$$

where a subscript ' $o$ ' represents the average over an index ( $m$ or $y)$. 
Here, we denote the interannual variance of $\epsilon_{y m}$ by

$$
\mathrm{V}\left(\epsilon_{\mathrm{ym}}\right)=\sigma_{\mathrm{m}}^{2}
$$

for each month $\mathrm{m}$. The intermonthly correlations, $\mathrm{C}\left(\epsilon_{y \mathrm{~m}}, \epsilon_{\mathrm{yn}}\right)$, are then defined as

$$
\mathrm{C}\left(\epsilon_{\mathrm{ym}}, \epsilon_{\mathrm{yn}}\right)=\frac{\mathrm{V}\left(\epsilon_{\mathrm{ym}}, \epsilon_{\mathrm{yn}}\right)}{\sigma_{\mathrm{m}} \sigma_{\mathrm{n}}}=\phi_{\mathrm{mn}}, \quad \mathrm{m}, \mathrm{n}=1,2,3, \quad \mathrm{~m} \neq \mathrm{n},
$$

where $\mathrm{V}\left(\epsilon_{y m}, \epsilon_{y n}\right)$ denotes the covariance between the intraseasonal components in months $m$ and $n$. The interannual variance of the intraseasonal component is then

$$
\begin{aligned}
V\left(\epsilon_{y o}\right) & =E\left[\left(\frac{1}{3} \sum_{m=1}^{3} \epsilon_{y m}\right)^{2}\right] \\
& =\frac{1}{9}\left(\sigma_{1}^{2}+\sigma_{2}^{2}+\sigma_{3}^{2}+2 \sigma_{1} \sigma_{2} \phi_{12}+2 \sigma_{2} \sigma_{3} \phi_{23}+2 \sigma_{1} \sigma_{3} \phi_{13}\right),
\end{aligned}
$$

where $\mathrm{E}$ denotes the expected value.

In order to estimate the terms on the right hand side of equation (5), we consider the expectation value of the monthly differences of $\epsilon_{y m}$ :

$$
\begin{aligned}
& E\left[\left(\begin{array}{c}
\epsilon_{y 1}-\epsilon_{y 2} \\
\epsilon_{y 2}-\epsilon_{y 3}
\end{array}\right)\left(\begin{array}{c}
\epsilon_{y 1}-\epsilon_{y 2} \\
\epsilon_{y 2}-\epsilon_{y 3}
\end{array}\right)^{\top}\right]= \\
& \left(\begin{array}{cc}
\sigma_{1}^{2}-2 \sigma_{1} \sigma_{2} \phi_{12}+\sigma_{2}^{2} & \sigma_{1} \sigma_{2} \phi_{12}-\sigma_{1} \sigma_{3} \phi_{13}-\sigma_{2}^{2}+\sigma_{2} \sigma_{3} \phi_{23} \\
\sigma_{1} \sigma_{2} \phi_{12}-\sigma_{1} \sigma_{3} \phi_{13}-\sigma_{2}^{2}+\sigma_{2} \sigma_{3} \phi_{23} & \sigma_{2}^{2}-2 \sigma_{2} \sigma_{3} \phi_{23}+\sigma_{3}^{2}
\end{array}\right) .
\end{aligned}
$$

From equation (1), differences in the monthly mean are assumed to be entirely due to the intraseasonal component (for example, $x_{y 1}-x_{y 2}=\epsilon_{y 1}-\epsilon_{y 2}$ ). Hence the monthly moments of $\epsilon_{y m}$ are defined in terms of the monthly moments of $x_{y m}$ :

$$
E\left(\epsilon_{y 1}-\epsilon_{y 2}\right)^{2}=E\left(x_{y 1}-x_{y 2}\right)^{2} \approx \frac{1}{Y} \sum_{y=1}^{Y}\left(x_{y 1}-x_{y 2}\right)^{2} \equiv a
$$




$$
\begin{aligned}
& E\left(\epsilon_{y 1}-\epsilon_{y 2}\right)\left(\epsilon_{y 2}-\epsilon_{y 3}\right)=E\left(x_{y 1}-x_{y 2}\right)\left(x_{y 2}-x_{y 3}\right) \\
& \quad \approx \frac{1}{Y} \sum_{y=1}^{Y}\left(x_{y 1}-x_{y 2}\right)\left(x_{y 2}-x_{y 3}\right) \equiv b, \\
& E\left(\epsilon_{y 2}-\epsilon_{y 3}\right)^{2}=E\left(x_{y 2}-x_{y 3}\right)^{2} \approx \frac{1}{Y} \sum_{y=1}^{Y}\left(x_{y 2}-x_{y 3}\right)^{2} \equiv c .
\end{aligned}
$$

From equation (6), this leads to the following set of equations

$$
\begin{aligned}
& \sigma_{1}^{2}-2 \sigma_{1} \sigma_{2} \phi_{12}+\sigma_{2}^{2} \approx \mathrm{a}, \\
& \sigma_{1} \sigma_{2} \phi_{12}-\sigma_{1} \sigma_{3} \phi_{13}-\sigma_{2}^{2}+\sigma_{2} \sigma_{3} \phi_{23} \approx \mathrm{b}, \\
& \sigma_{2}^{2}-2 \sigma_{2} \sigma_{3} \phi_{23}+\sigma_{3}^{2} \approx \mathrm{c} .
\end{aligned}
$$

\subsection{Solution for Australian surface temperature}

Equations (10), (11) and (12) represent three equations with six unknowns. In order to use monthly mean data, it is necessary to make some assumptions about the monthly statistics of the climate data. For Australian surface temperature, it is assumed that the standard deviation of $\epsilon_{y m}$ varies linearly across a season by a parameter $\beta$ :

$$
\sigma_{1}=\sigma_{2}-\beta, \quad \sigma_{3}=\sigma_{2}+\beta, \quad|\beta|<\sigma_{2} .
$$

It is also assumed that the intermonthly correlations of $\epsilon_{y m}$ are stationary within each season:

$$
\phi_{12}=\phi_{23} .
$$

Grainger et al. [3] showed that the assumptions in equations (13) and (14) to a first order account for the variability of Australian surface temperature within a season. Recognising that day-to-day weather events are unpredictable beyond a week or two, we further assume that the intraseasonal components are uncorrelated if they are a month or more apart:

$$
\phi_{13}=0 \text {. }
$$


Since daily surface temperature is a first order autoregressive process, Zheng et al. [8] showed that the estimation error is reduced by applying the constraint

$$
0 \leq \phi_{12} \leq 0.1
$$

Applying equations (13), (14) and (15) to equations (10), (11) and (12) leads to the following set of three equations with three unknowns:

$$
\begin{aligned}
& \left(\sigma_{2}-\beta\right)^{2}-2 \sigma_{2}\left(\sigma_{2}-\beta\right) \phi_{12}+\sigma_{2}^{2} \approx \mathrm{a}, \\
& \sigma_{2}^{2}\left(2 \phi_{12}-1\right) \approx \mathrm{b}, \\
& \left(\sigma_{2}+\beta\right)^{2}-2 \sigma_{2}\left(\sigma_{2}+\beta\right) \phi_{12}+\sigma_{2}^{2} \approx \mathrm{c} .
\end{aligned}
$$

Equations (17), (18) and (19) are solved by rearranging to form a cubic equation for $\phi_{12}$. The real root of the solution is then constrained by equation (16) before back-substitution into equations (17) and (19) to find $\beta$ and $\sigma_{2}$. The intraseasonal variance is then estimated as

$$
\widehat{\nabla}\left(\epsilon_{\text {yo }}\right)=\frac{1}{9}\left[\hat{\sigma}_{2}^{2}\left(3+4 \hat{\phi}_{12}\right)+2 \hat{\beta}^{2}\right],
$$

where the caret ${ }^{\wedge}$ denotes an estimate.

\subsection{Potential predictability}

The total interannual variability of the seasonal mean of a climate variable is estimated by the sample variance

$$
\widehat{\nabla}\left(x_{y_{0}}\right)=\frac{1}{Y-1} \sum_{y=1}^{Y}\left[x_{y o}-x_{o o}\right]^{2} .
$$

A commonly used definition of potential predictability (for example, Frederiksen and Zheng [2], Zheng et al. [9]) is the fraction of total interannual 
variability of the seasonal mean that is not due to the intraseasonal variance,

$$
\text { Potential Predictability } \equiv 1-\frac{\widehat{\nabla}\left(\epsilon_{\text {yo }}\right)}{\widehat{\nabla}\left(x_{\text {yo }}\right)},
$$

which is calculated using equations (20) and (21).

\section{Potential predictability of Western Australian surface temperature}

To illustrate the methodology, the potential predictability of surface temperature for Western Australia is estimated using equation (22). Monthly mean surface maximum and minimum temperature were obtained from the Australian Bureau of Meteorology National Climate Centre gridded historical dataset [4]. Surface maximum and minimum temperature were chosen for analysis since they are direct observations from the network of Australian weather stations. Data was obtained for period November 1950 to December 2000 on a grid of $1^{\circ} \times 1^{\circ}$ latitude/longitude land surface points.

The estimated potential predictability of Western Australian surface maximum temperature for all 12 three month 'seasons' is shown in Figure 1 as a percentage. Each season is denoted by the first letter of each month (for example, December-January-February is DJF). North of $20^{\circ} \mathrm{S}$, there is generally high potential predictability (at least 50\%) throughout most of the year. However, in other regions, there is a more pronounced seasonal cycle. For example, potential predictability along the west coast goes from a maximum (over 60\%) in JJA to a minimum (less than 20\%) in DJF.

Figure 2 shows the estimated potential predictability of Western Australian surface minimum temperature. Like surface maximum temperature, the potential predictability is generally high north of $20^{\circ} \mathrm{S}$, although it is 


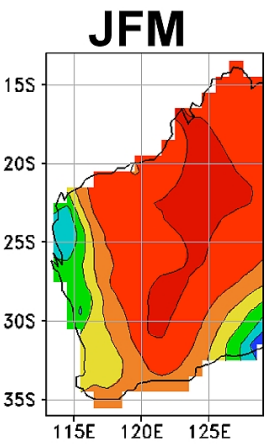

MJJ

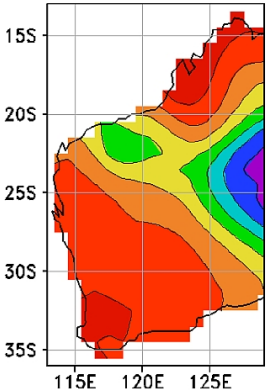

SON

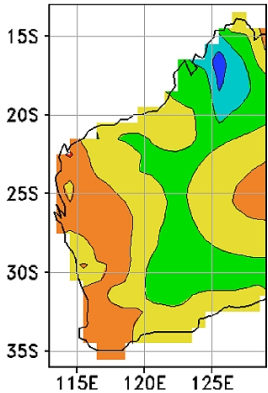

FMA

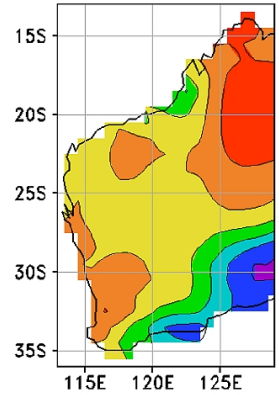

JJA

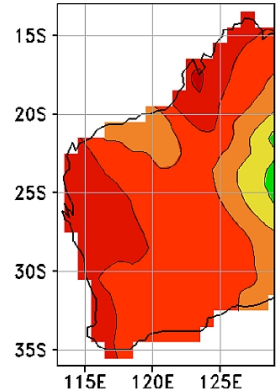

OND

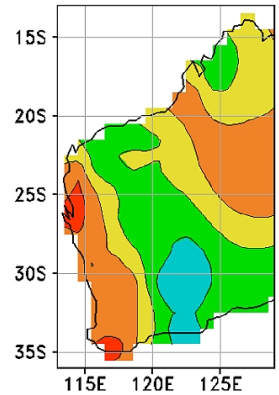

MAM

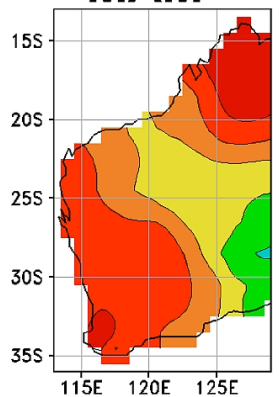

JAS

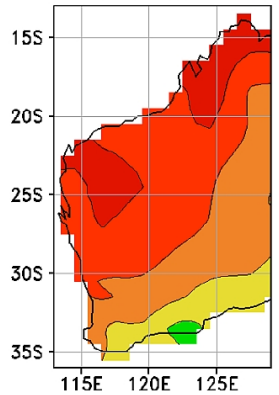

NDJ

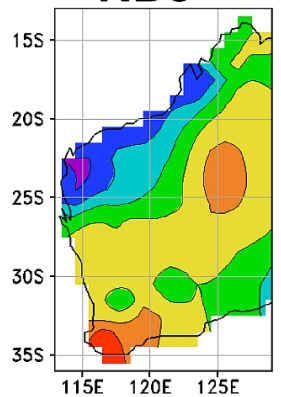

AMJ

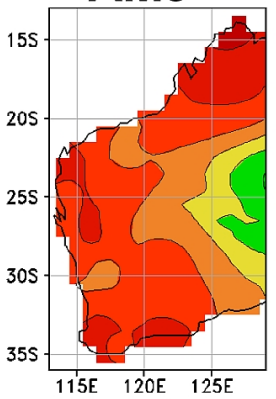

ASO

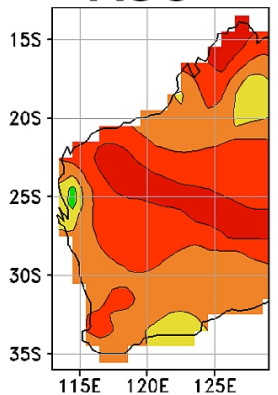

DJF

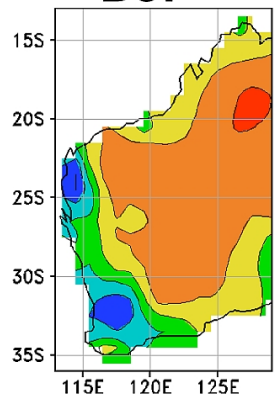

80

70

60

50

40

30

20

10

0

FiguRE 1: Estimated potential predictability for Western Australian surface maximum temperature for the period 1951-2000, shown as a percentage. 


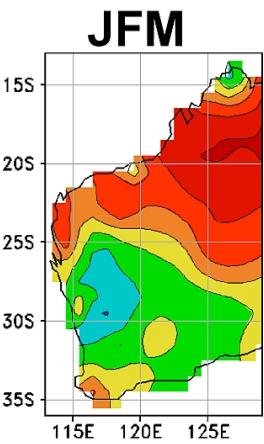

MJJ

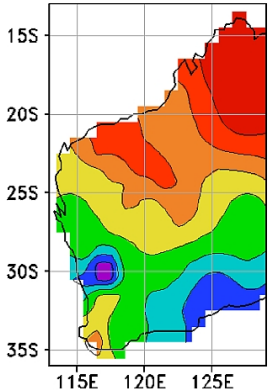

SON

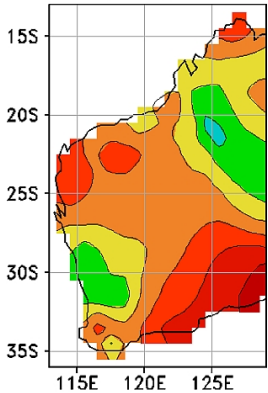

FMA

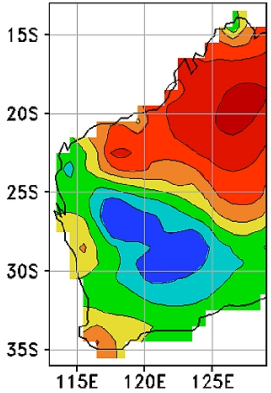

JJA

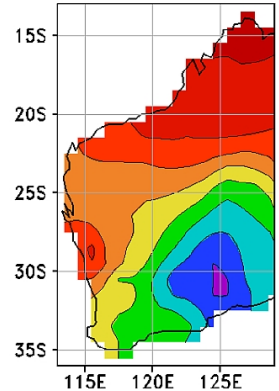

OND

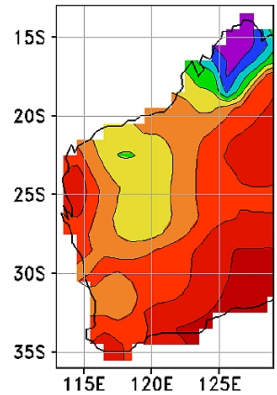

MAM

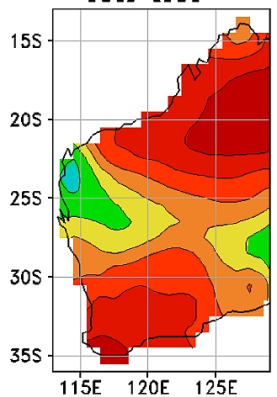

JAS

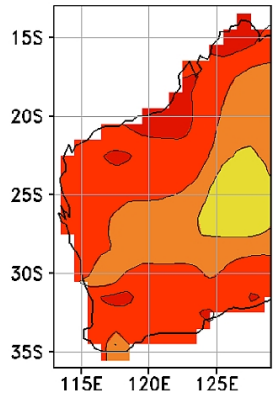

NDJ

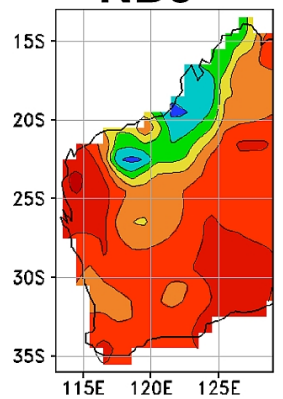

AMJ

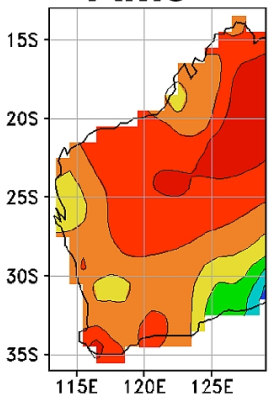

ASO

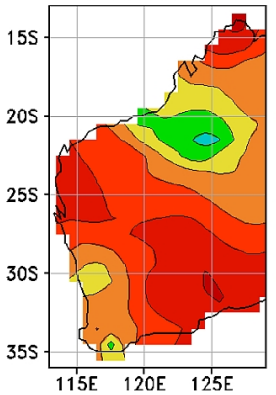

DJF

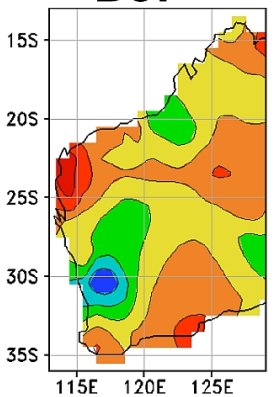

80

70

60

50

40

30

20

10

0

FiguRE 2: Estimated potential predictability for Western Australian surface minimum temperature for the period 1951-2000, shown as a percentage. 
much lower in OND. Elsewhere, seasonal cycles can be seen, but the structure and season of these cycles differ from surface maximum temperature. For example, along the south coast potential predictability goes from less than $20 \%$ in JJA to over $70 \%$ in OND. The different seasonal cycles are likely to be due to differences in the processes involved with daily surface maximum and minimum temperature, which have been considered in terms of the diurnal cycle of the surface energy budget [6].

\section{Conclusions}

An existing Analysis of Variance method has been extended to estimate the intraseasonal variance of the seasonal mean of a climate variable in all seasons. By making assumptions about the monthly statistics that are valid for Australian surface temperature, the intraseasonal variance, and therefore the potential predictability, is estimated from monthly mean data for all seasons. The new methodology was applied to surface maximum and minimum temperature for Western Australia for the period 1951-2000. The potential predictability shows distinct cycles over the 12 three month seasons. These cycles are different for surface maximum and minimum temperature, which is likely to be due to differences in the physical processes involved for the daily temperature. In general, the potential predictability for surface minimum temperature is somewhat higher than for surface maximum temperature. The sources of potential predictability will be examined in future by extending to all seasons the current methodology for estimating the intraseasonal covariance of two climate variables [7].

Acknowledgements Monthly mean surface maximum and minimum temperature data for Western Australia was provided by the National Climate Centre of the Australian Bureau of Meteorology. SG is supported by the Australian Climate Change Science Program of the Australian Department 
of Climate Change. XZ is supported by the New Zealand Foundation for Research, Science and Technology (contract C01x0701). CSF acknowledges the support of the Western Australian Department of Environment and Conservation under the Indian Ocean Climate Initiative Stage 3.

\section{References}

[1] R. J. B. Fawcett, D. A. Jones and G. S. Beard. A verification of publicly issued seasonal forecasts issued by the Australian Bureau of Meteorology: 1998-2003. Aust. Meteor. Mag., 54:1-13, 2005. C599

[2] C. S. Frederiksen and X. Zheng. Coherent Structures of Interannual Variability of the Atmospheric Circulation: The Role of Intraseasonal Variability. Frontiers in Turbulence and Coherent Structures, World Scientific Lecture Notes in Complex Systems, Vol. 6, Eds Jim Denier and Jorgen Frederiksen, World Scientific Publications, 87-120, 2007. C599, C600, C603

[3] S. Grainger, C. S. Frederiksen and X. Zheng. Estimating the potential predictability of Australian surface maximum and minimum temperature. Climate Dynamics, Accepted, 2008. C602

[4] D. A. Jones and B. C. Trewin. The spatial structure of monthly temperature anomalies over Australia. Aust. Meteor. Mag., 49:261-276, 2000. C604

[5] E. N. Lorenz. On the existence of extended range predictability. J. Appl. Meteor., 12:543-546, doi:10.1175/1520-0450(1973)012ز0543:OTEOER ¿2.0.CO;2, 1973. C600

[6] I. G. Watterson. The diurnal cycle of surface air temperature in simulated present and doubled CO2 climates. Climate Dynamics, 13:533-545, doi:10.1007/s003820050181, 1997. C607 
[7] X. Zheng and C. S. Frederiksen. Variability of seasonal-mean fields arising from intraseasonal variability. Part 1, methodology. Climate Dynamics, 23:177-191, doi:10.1007/s00382-004-0428-7, 2004. C600, C607

[8] X. Zheng, H. Nakamura and J. A. Renwick. Potential predictability of seasonal means based on monthly time series of meteorological variables. J. Climate, 13:2591-2604, doi:10.1175/1520-0442(2000)013¡2591:PPOSMB ¿2.0.CO;2, 2000. C603

[9] X. Zheng, M. Sugi and C. S. Frederiksen. Interannual variability and predictability in an ensemble of climate simulations with the MRI-JMA AGCM. J. Meteor. Soc. Jap., 82:1-18, doi:10.2151/jmsj.82.1, 2004. C600, C603

\section{Author addresses}

1. S. Grainger, Centre for Australian Weather and Atmospheric Research, Bureau of Meteorology, GPO Box 1289, Melbourne, Victoria 3001, Australia. mailto:s.grainger@bom.gov . au

2. C. S. Frederiksen, Centre for Australian Weather and Atmospheric Research, Bureau of Meteorology, GPO Box 1289, Melbourne, Victoria 3001, Australia.

3. X. Zheng, National Institute of Water and Atmospheric Research, Wellington, NEW ZEALAND 